\title{
Drug - related emergency department visits by elderly patients presenting with non-specific complaints
}

Christian H Nickel ${ }^{1 *+}$, Juliane M Ruedinger ${ }^{1+}$, Anna S Messmer ${ }^{1}$, Silke Maile ${ }^{1}$, Arno Peng ${ }^{1}$, Michael Bodmer ${ }^{1,2}$, Reto W Kressig ${ }^{3}$, Stephan Kraehenbuehl ${ }^{2}$ and Roland Bingisser ${ }^{1}$

\begin{abstract}
Background: Since drug-related emergency department (ED) visits are common among older adults, the objectives of our study were to identify the frequency of drug-related problems (DRPs) among patients presenting to the ED with non-specific complaints (NSC), such as generalized weakness and to evaluate responsible drug classes.

Methods: Delayed type cross-sectional diagnostic study with a prospective 30 day follow-up in the ED of the University Hospital Basel, Switzerland. From May 2007 until April 2009, all non-trauma patients presenting to the ED with an Emergency Severity Index (ESI) of 2 or 3 were screened and included, if they presented with non-specific complaints. After having obtained complete 30-day follow-up, two outcome assessors reviewed all available information, judged whether the initial presentation was a DRP and compared their judgment with the initial ED diagnosis. Acute morbidity ("serious condition") was allocated to individual cases according to predefined criteria.

Results: The study population consisted of 633 patients with NSC. Median age was 81 years (IQR 72/87), and the mean Charlson comorbidity index was 2.5 (IQR 1/4). DRPs were identified in 77 of the 633 cases (12.2\%). At the initial assessment, only 40\% of the DRPs were correctly identified. 64 of the 77 identified DRPs (83\%) fulfilled the criteria "serious condition". Polypharmacy and certain drug classes (thiazides, antidepressants, benzodiazepines, anticonvulsants) were associated with DRPs.
\end{abstract}

Conclusion: Elderly patients with non-specific complaints need to be screened systematically for drug-related problems.

Trial registration: ClinicalTrials.gov:NCT00920491

\section{Background}

Drug-related ED visits are common. Up to $25 \%$ of ED consultations by elderly patients are due to drug-related problems (DRPs), depending on the definitions of DRP used [1-6]. Older patients are more frequently affected by DRPs than younger ones $[1,2,7]$. Contributing factors are physiologic changes associated with aging, which include impaired renal and hepatic function, as well as decreased total body water and lean body mass [8]. Additionally, older adults use more medications because of the co-existence of multiple comorbidities $[9,10]$. As a

\footnotetext{
* Correspondence: nickelc@uhbs.ch

†Equal contributors

${ }^{1}$ Department of Emergency Medicine, University Hospital Basel, Basel,

Switzerland

Full list of author information is available at the end of the article
}

consequence, polypharmacy is highly prevalent in the older patient [11]. Furthermore, the presence of dementia or confusion may lead to patient errors due to complex medication regimens, resulting in DRPs $[12,13]$.

Emergency Physician recognition of DRPs appears to be dependent on the mode of presentation [14]. Patients with DRPs can present to the ED with specific symptoms, e.g. rash, bleeding, arrhythmias, or hypoglycemia $[15,16]$. However, patients with DRPs may also present with non-specific complaints (NSC), such as generalized weakness which may make a DRP more difficult to be identified, considering the fact that ED physicians are failing to identify DRPs in up to $40 \%$ even in a general ED population [3]. 
Current research on DRPs in older patients is focused on falls and delirium $[17,18]$, but up to $20 \%$ of elderly patients present to the ED with NSC, and the underlying cause of their symptoms is often not known $[19,20]$. Importantly, patients with NSC are at risk of adverse health outcomes [21-23]; probably because of their often older age, cognitive and functional impairment, multiple comorbidities, and sub-acute or atypical presentation of acute illness $[19,24,25]$. This patient group with NSC poses significant challenges to emergency physicians (EPs), as the differential diagnostic spectrum is so wide, ranging from lack of social support to life-threatening disease $[26,27]$. The need for research and training in this area has been addressed $[28,29]$.

Currently it is not known how many patients with NSC suffer from DRPs. DRPs represent an important, potentially preventable and curable condition [30]. It can be speculated that DRPs in patients with NSC may be overlooked. Therefore, the objectives of our study were to identify the frequency of DRPs among patients presenting to the ED with NSC, to evaluate drugs and classes being associated with such DRPs and to assess the proportion of missed DRPs in the initial assessment. An additional aim was to determine the frequency of DRPs associated with acute morbidity.

\section{Methods}

\section{Study design}

This present study is a predefined part of the prospective Basel-non-specific complaints (BANC) study [22] with diagnostic assessment after a 30 day follow-up period by a panel of experts, representing a delayed type crosssectional study [31]. The study protocol was approved by the local ethics committee (www.ekbb.ch, Reference Number EKBB 73/07) and it is registered with Clinical Trials (www.clinicaltrials.gov, NCT00920491). It is in compliance with the Helsinki Declaration.

\section{Study setting and population}

The study was carried out in the ED of the University Hospital Basel, Switzerland. The hospital is an urban 700-bed tertiary care center with an ED census of over 41'000 patients per year. From May 24th 2007 until April 15th 2009, all non-trauma patients 18 years of age or older with an Emergency Severity Index (ESI) level of 2 or 3 [32] presenting to the ED were consecutively screened for inclusion. The ESI, a 5-level triage tool with proven reliability and validity for the German translation [33] was used in order to exclude all patients with lifethreatening conditions (ESI 1), as well as patients with conditions in which a full work-up was not intended ("see-and-treat" pathway, ESI 4 and 5). All ED patients were screened twice for inclusion: First by a triage-nurse in order to include all ESI 2 and ESI 3 patients and secondly each resident on duty screened all ESI 2 and ESI 3 patients for their presenting symptoms using a one-page screening tool. Enrolment was then carried out by 3 previously trained study physicians.

\section{Inclusion criteria}

Patients were included if they presented with NSC. NSCs are defined as the entity of complaints which are not part of the set of specific complaints or signs or where an initial working diagnosis cannot be definitively established [22]. For such complaints, no evidence-based management protocols (EPs) exist. Typical examples of NSC are "generalized weakness", "general deterioration" or "lack of community support" [34,35].

\section{Exclusion criteria}

Patients with specific complaints (e.g. syncope, chest pain) were not included. Hemodynamically unstable patients or with vital parameters significantly out of the normal range (systolic blood pressure $<80$ or $>180 \mathrm{mmHg}$, respiration rate $>20 / \mathrm{min}$, tympanal body temperature $>38.5^{\circ} \mathrm{C}$, $\left.\mathrm{SpO}_{2}<92 \%\right)$ were also excluded. Patients with abnormal vital signs can be considered as "specific", since work-up is generally straight-forward, for example for shock, even when the symptoms are non-specific. In addition, patients with known terminal conditions as well as patients referred from other hospitals were not eligible for inclusion.

\section{Measurements}

Demographic baseline data, ESI, all complaints (using a structured interview form), vital signs (pulse, blood pressure, respiratory rate, and oxygen saturation), Glasgow Coma Scale, medical history, physical examination, and electrocardiography reading were obtained on arrival and registered on the patient's case report form. Additionally, the body mass index (BMI) was calculated. For quantification of comorbidities the Charlson Comorbidity Index was used [36]. This tool has been validated for population-based studies to estimate the risk of death. Lab testing was done in all patients and, in the vast majority, chest X-ray and urinalysis was performed. Treatment was initiated at the discretion of the ED physician in charge.

\section{Assessment of medication}

We assessed all drugs reported by the patient during a bedside interview, or reported by proxies or by the family physician, or found in our electronic patient records. Drugs were grouped into predefined pharmacological classes (Table 1). Additionally, we analyzed the drugs recorded according to the Beers criteria, a list of potentially inappropriate medications in older adults which aims to minimize drug-related problems [37]. The Beers criteria are regularly updated [38] and based on 
Table 1 Prescribed drugs at presentation to the ED

\begin{tabular}{|c|c|c|}
\hline Drug class & $\begin{array}{c}\text { Number } \\
\text { of patients }\end{array}$ & $\begin{array}{c}\text { Percent \% } \\
\text { (total } n=633 \text { ) }\end{array}$ \\
\hline Diuretics (all types) & 294 & 46.4 \\
\hline Non-thiazide diuretics & 225 & 41.4 \\
\hline Thiazide diuretics & 136 & 21.5 \\
\hline ACE inhibitors/AR blockers & 231 & 36.5 \\
\hline Beta-blockers & 211 & 33.3 \\
\hline Other cardiovascular drugs ${ }^{\dagger}$ & 129 & 20.4 \\
\hline Platelet aggregation inhibitors & 236 & 37.3 \\
\hline Vitamin K- antagonists & 90 & 14.2 \\
\hline Systemic steroids & 47 & 7.4 \\
\hline NSAID & 98 & 15.5 \\
\hline Opioids & 69 & 10.9 \\
\hline All psychotropic drugs & 298 & 47.1 \\
\hline Benzodiazepines & 121 & 19.1 \\
\hline Neuroleptics & 91 & 14.4 \\
\hline Antidepressants & 160 & 25.3 \\
\hline Other $^{\ddagger}$ & 86 & 13.6 \\
\hline Anticonvulsants & 61 & 9.6 \\
\hline Anti-Parkinson drugs & 48 & 7.6 \\
\hline
\end{tabular}

${ }^{\dagger}$ calcium channel blockers, nitrates, digoxine, amiodarone.

${ }^{\ddagger}$ other hypnotica such as barbiturates, zopiclone and zolpidem and others, such as St. Johns Worth.

expert consensus. They are developed through literature review and a questionnaire. They have been evaluated by recognized experts in geriatric care, clinical pharmacology and psychopharmacology.

\section{Patient follow up and endpoint ascertainment}

Written 30-day follow-up data was obtained from hospital discharge reports or the patients' primary care physicians (questionnaire). Outcome assessors reviewed all available medical records and data of the study patients from the time of ED presentation to 30-day follow-up. Six ED physicians one of whom is a clinical pharmacologist, all certified in internal medicine and experienced in emergency medicine (outcome assessors) were available for outcome ascertainment. In each session, two outcome assessors, reviewed all discharge records and established a final "gold standard" diagnosis according to the 10th International Classification of Diseases and Related Health Problems (ICD-10). Furthermore, the diagnoses were evaluated to be drug-related or not using Micromedex [39]. For each medication entered, a detailed list of possible DRPs was produced using Micromedex. Where needed, a board-certified clinical pharmacologist was consulted. In the 22 cases of disagreement between outcome assessors, cases were reviewed and adjudicated in conjunction with the BANC expert panel, consisting of two physicians certified in internal medicine with at least 10 years of clinical experience as previously described [22].

In a second step, the expert panel classified the DRP according to the Pharmaceutical Care Network Europe (PCNE) Classification (see below) [40].

The DRPs identified as described above were compared with the ED diagnoses given on the day of the visit. By comparison, the proportion of missed DRPs could be identified.

\section{Definition and classification of drug-related problems (DRPs)}

According to the PCNE Classification V 5.01, DRPs are defined as "events or circumstances involving drug therapy actually or potentially interfering with desired health outcomes" [40]. In this classification, DRPs can be assigned to six main categories, so-called "primary domains" P1-P6: (P1) adverse drug reaction (allergic, nonallergic or toxic side-effects), (P2) drug choice problem (patient gets or is going to get a wrong (or no drug) drug for his/her disease and/or condition), (P3) dosing problem (patient gets more or less than the amount of drug he/she requires), (P4) drug use problem (wrong or no drug taken/administered), (P5) drug-drug interactions, and (P6) others. We chose the PCNE classification, since it has been shown to contain all required aspects to describe and classify DRPs for research and practice purpose [41]. Furthermore, it has been evaluated in our institution and is considered to be a practical tool in the hospital setting [42]. Polypharmacy was defined as the prescription of 6 or more drugs at presentation [43].

\section{Serious condition}

A "serious condition" was attributed to individual cases using predefined criteria that are covered in a comprehensive list [22]. Briefly, a "serious condition" was defined as a potentially life-threatening condition, or any condition requiring an early intervention (e.g. central nervous dysfunction due to hyponatremia) to prevent health status deterioration leading to potential morbidity or death within 30 days of the initial ED presentation.

\section{Data analysis}

In case of categorical variables, crosstables were calculated. In case of ordinal or metric variables, mean, median, standard deviation, minimum, maximum were calculated. To predict the influence of the number of concomitantly prescribed drugs and of certain drug classes (e.g. diuretics, psychotropic drugs) on the outcomes DRP or DRP with "serious condition", a logistic regression model was chosen. Adjustment for age, gender and comorbidities was performed by adding these cofactors to the regression model. Results are presented as two-sided 
p-values and odds ratios (ORs) with their corresponding $95 \%$ confidence intervals. In the case of continuous or ordinal predictors, the OR is expressed as the ratio of the odds increasing the predictor from the 1rd to the 3st quartile (25 and 75 percentile, respectively), representing a typical above average to a typical below average value. A $\mathrm{p}$-value $<0.05$ was considered significant. This study is exploratory; therefore p-values were not adjusted for multiple comparisons. All analyses were done using $\mathrm{R} v \mathrm{v}$ 2.8.0 (A Language and Environment for Statistical Computing).

\section{Results}

From May 24th 2007 until April 15th 2009, 22'782 nontrauma patients presented to the ED. 9926 patients were triaged as ESI level 2 or ESI 3 and therefore screened for inclusion into the current study. Of these, 714 patients (7.2\%) presented with NSC and were consecutively enrolled in our study (see Figure 1). During post-hoc case reviews, the BANC expert panel recalled the inclusion of 81 patients due to the presence of exclusion criteria, mostly due to the presence of specific complaints. The final study population consisted of 633 patients with NSC. No patient was lost to follow-up. Baseline characteristics of the study population are presented in Table 2. Median age was 81 years (IQR 72/87), $62.6 \%$ of subjects were female, median BMI was $23.4 \mathrm{~kg} / \mathrm{m}^{2}$ (IQR 20.4/26.4). Mean Charlson comorbidity index was 2.5 (IQR 1/4). 581 patients $(91.7 \%)$ took 1 or more medications (prescribed, or over the counter). The number of medications taken ranged from 0 to 17 with a median number of 5 drugs (IQR 3/8). The detailed analyses of the drug categories are presented in Table 1.

A total number of 77 patients with DRPs were identified, attributing to $12.2 \%$ of the total study population (633 patients). A "Serious Condition" as defined above was detected in 64 of the 77 identified DRPs $(83 \%)$. Of the 633 patients with NSC screened for DRPs, 387 suffered from a "serious condition" (61\%). The comparison of the 77 cases with a DRP with the initial ED diagnosis given on the day of the visit revealed that 48 cases with a DRP (60\%) were initially not identified. Most commonly missed drug-related diagnoses were electrolyte disorders (hyponatremia and others, 20 cases), hypovolemia (7 cases) and intoxications (5 cases).

42 DRPs (56\% of all DRPs) were classified under the P1 domain "adverse drug reactions (ADR)". Nine DRPs (12\% were classified as P2 (drug choice problem), 16 (21\%) as P3 (dosing problems), 2 (3\%) as P4 (drug use

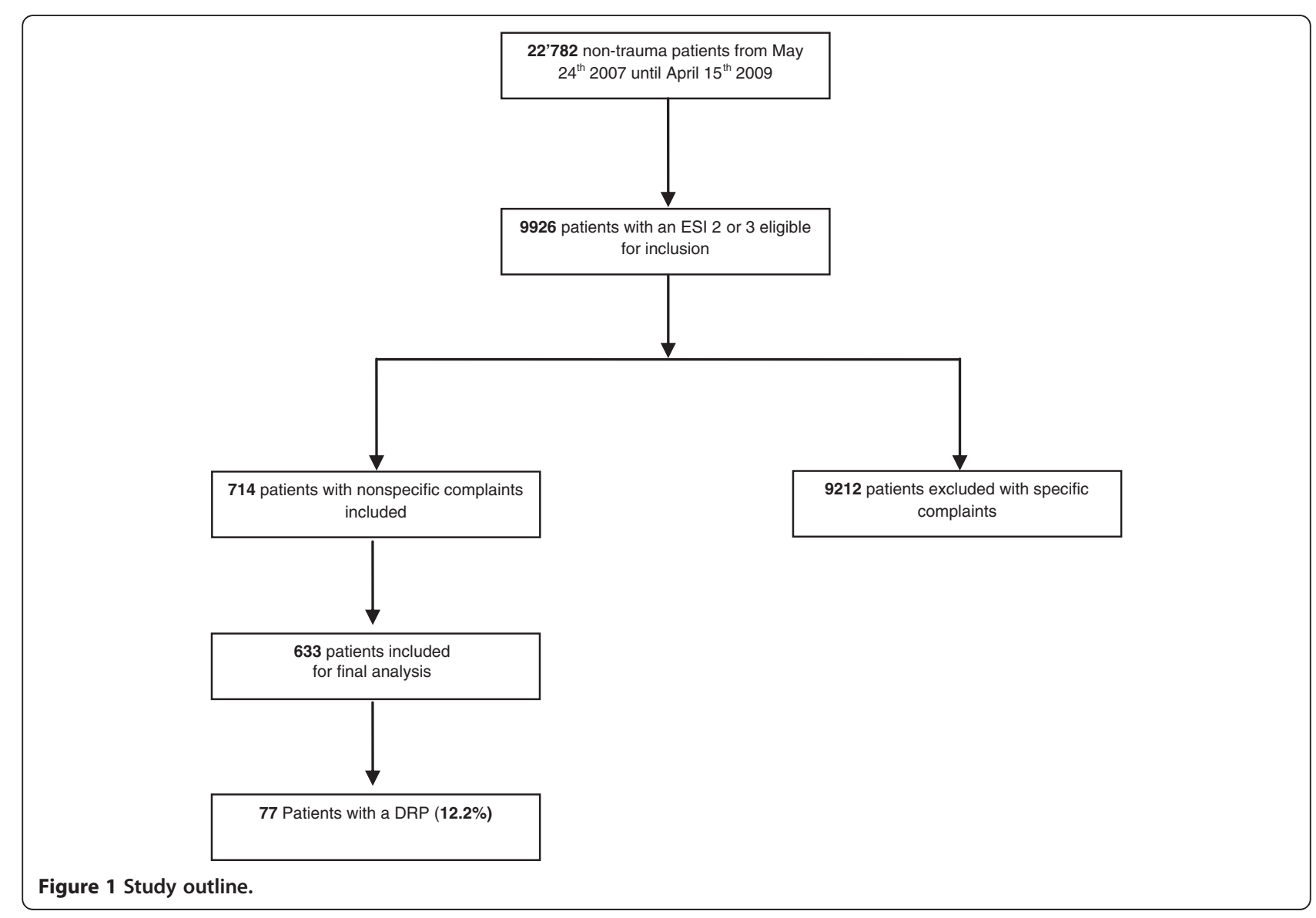


Table 2 Baseline characteristics of the study population

\begin{tabular}{lc}
\hline Characteristics & $\begin{array}{c}\text { Total population } \\
\text { (N = 633) }\end{array}$ \\
\hline Age, median (IQR) & $81(72 / 87)$ \\
Female (\%) & $396(62.6)$ \\
BMI, median (IQR) & $23.4(20.4 / 26.4)$ \\
Living situation: & \\
$\quad$ - independent (\%) & $145(22.9)$ \\
$\quad$ - with family help (\%) & $208(32.9)$ \\
$\quad$ - with home care (\%) & $214(33.8)$ \\
$\quad$ - nursing home (\%) & $66(10.4)$ \\
Charlson Comorbidity Index, median (IQR) & $2(1 / 4)$ \\
Number of concomitant drugs, median (IQR) & $5(3 / 8)$ \\
Patients with serious condition, number (\%) & $387(61.1)$ \\
Patients with DRP, number (\%) & $77(12.2)$ \\
Patients with DRP as serious condition, number (\%) & $64(83.1)$ \\
\hline
\end{tabular}

$\mathrm{IQR}=$ Inter-Quartile Range.

$B M I=$ Body Mass Index.

$M D R D=$ Modification of Diet in Renal Disease, equation to predict glomerular filtration rate from serum creatinine [44].

$D R P=$ drug-related problems, as defined in the PCNE-Classification V5.1.

problems), 3 (4\%) as P5 (drug interactions) and $3(4 \%)$ as P6 (other) (see Figure 2).

\section{Drugs associated with DRP}

In our population, each additional drug accounted for an increase of $10 \%$ in the probability of suffering from a DRP. This association was observed for treatment with 3 to 8 drugs.

In patients presenting with NSC, the use of psychotropic drugs was significantly associated with DRPs (adjusted OR 2.32, 95\% CI $1.39-3.88, \mathrm{p}=0.001$ ). This association became stronger with an increasing number

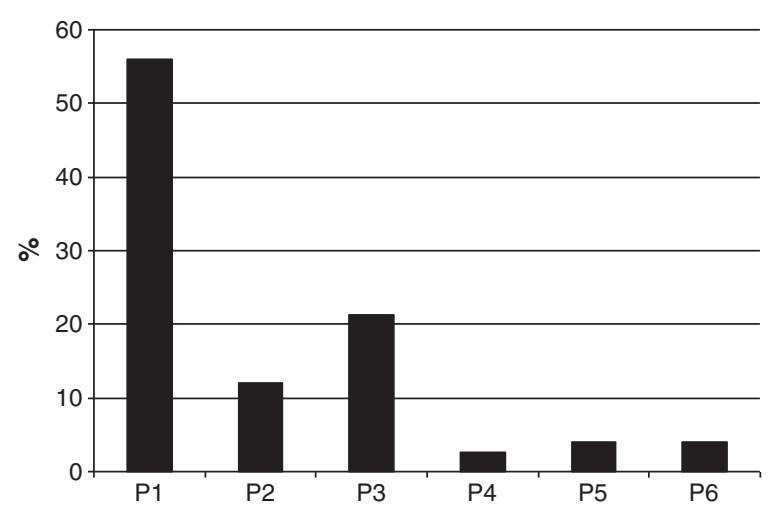

Figure 2 PCNE Classification and percentage of all our identified drug-related problems (DRP), $\mathbf{N}=77$. It has six primary domains for problems: $\mathrm{P} 1=$ adverse drug reactions, $\mathrm{P} 2=$ Drug choice problem, $\mathrm{P} 3=$ Dosing problem, $\mathrm{P} 4=$ Drug use problem, P5 $=$ Interactions, $\mathrm{P} 6=$ other . of the psychotropic drugs used (adjusted OR for one psychotropic drug.1.89, 95\% CI $1.06-3.37, \mathrm{p}<0.05$ and for 2 psychotropic drugs 2.95, 95\% CI $1.51-5.76$, $\mathrm{p}<0.01$ ). Both the intake of benzodiazepines and antidepressants were associated with DRPs $(\mathrm{p}<0.001)$ in patients with NSC with an adjusted OR of 2.5 (95\% CIs 1.47 - 4.28) and 2.41 (1.46 - 3.98), respectively. Furthermore the use of anticonvulsants (adjusted OR 3.06, 95\% CI $1.55-6.02, \mathrm{p}<0.05)$ and of diuretics (adjusted OR 1.8, 95\% CI $1.06-3.04, \mathrm{p}<0.05$ ) was associated with an increased risk for DRPs. Further analysis of the risk associated with diuretics showed that the use of non-thiazide diuretics did not increase the risk for DRPs significantly (adjusted OR 1.54, 95\% CI $0.92-2.59, \mathrm{p}=0.1)$, but that the risk for DRPs was increased with the use of thiazides (adjusted OR 2.09; 95\% CI 1.21-3.62, p = 0.009) (see Table 3). In 7 cases, more than one drug was found to be associated with the respective DRP. Other drug-classes (e.g. NSAIDs, betablockers, neuroleptics, opioids) were not significantly associated with DRPs in our study population with NSC (data not shown).

\section{Corresponding final diagnosis}

The most frequent underlying causes of DRPs with NSC were hyponatremia (27\%) and drug-overdose (20\%). Hyponatremia was most commonly due to thiazides as well as due to the syndrome of inappropriate secretion of antidiuretic hormone (SIADH) associated with antidepressants or anticonvulsants. Drug-overdose was mostly due to prescribed benzodiazepines or opiates. A more detailed list of the corresponding final diagnosis is shown in Table 3.

\section{Discussion}

To our knowledge, this is the first study assessing DRPs in elderly ED patients with non-specific complaints. Our results show a prevalence of $12.2 \%$ for DRPs in this patient group. Thus, DRPs rank among the top five causes for non-specific ED presentations, with the vast majority (83\%) causing acute morbidity, classified as a "serious condition". This illustrates the importance of early detection of DRPs in the ED. In our study, 60\% of DRPs in patients visiting the ED with NSC were initially not diagnosed as medication-related, and were also not considered in the differential diagnosis.

The risk for a DRP increased with the number of prescribed medications and with treatment with certain drug classes, in particular antidepressants, benzodiazepines and anticonvulsants. Intake of thiazides, benzodiazepines, antidepressants and anticonvulsants was associated with a significantly increased risk for DRPs. The corresponding final diagnosis was most commonly hyponatremia or medication-overdose. 


\section{Table 3 Observed DRPs}

\begin{tabular}{ll}
\hline Involved organ system & Involved drugs $^{\dagger}$ \\
\hline $\begin{array}{l}\text { Electrolytes } \\
\text { Hyponatremia }\end{array}$ & $\begin{array}{l}\text { Thiazide diuretics (14), Non-thiazide } \\
\text { diuretics (2), Anticonvulsants (2), } \\
\text { Antidepressants }{ }^{ \pm} \text {(2), ACE/ARB } \\
\text { blockers (1) }\end{array}$ \\
Nypokalemia & $\begin{array}{l}\text { Non-thiazide diuretics (3), Thiazide } \\
\text { diuretics (1) }\end{array}$ \\
Hyperkalemia & ACE/ARB blockers (1) \\
Cardiovascular & \\
Bradycardia & Beta blockers (1) \\
Hypotension & Beta blockers (1) \\
Acute heart failure & NSAID (1) \\
Hypovolemia/orthostatic & Thiazide diuretics (7), Non-thiazide \\
dysregulation & diuretics (4),
\end{tabular}

\section{Kidney}

Hypovolemia, prerenal azotemia

Acute kidney injury

Coagulation system

Intracranial hemorrhage

\section{Gastrointestinal}

Nausea

Gastric ulcer/Gastritis

Central nervous system

Aggravation of Parkinson's

Status epilepticus

Hematology

Neutropenic complication

Anemia

\section{Endocrine}

Diabetes mellitus

Addison

Other

Overdosing

Falls

Tumor lysis syndrome

Abusus

${ }^{\dagger}$ In 7 cases, the DRP was caused by more than one drug class. In 2 cases, one drug caused more than one DRP.

${ }^{ \pm}$SSRI, SNRI, Tricyclic antidepressants.

${ }^{\ddagger}$ Vital signs within normal range at presentation.
Non-specific complaints are a common mode of presentation in the ED and have previously been described using terms, such as "general deterioration", "loss of energy", "weakness" or "home care impossible" [19,20,34,45]. Elderly patients with comorbidities, who are not institutionalized, belong to the high-risk population developing DRPs $[4,12,46]$. This risk group is highly represented in our study population. The vast majority of our patients with NSCs were not institutionalized (89.6\%) and had a relatively high burden of comorbidity compared to similar populations described [47,48].

It has been previously shown that for an elderly ED population the most common drugs causing DRPs are diuretics, oral anticoagulants, NSAIDs, antiarrhythmics, antiplatelet agents and psychotropic drugs [49]. In another study on older patients, Warfarin, Insulin, oral antiplatelet agents and oral hypoglycemic agents were implicated alone or in combination in 67\% of emergency hospitalizations for adverse drug events [50]. However, drug classes such as anticoagulants, NSAIDs, antiarrhythmics, and antiplatelet drugs tend to cause specific symptoms (e.g. bleeding, syncope) or signs (e.g. bradycardia, hypotension) in contrast to diuretics. This may explain the under-representation of DRPs induced by these substances in our study population whose chief complaints were merely non-specific.

In accordance with the Beers criteria, we observed several cases of DRPs due to SSRIs, benzodiazepines and anticonvulsants [37]. However we also detected DRPs associated with drugs which are neither listed in the Beers criteria of 2003 nor 2012, e.g. thiazide-diuretics [38]. This discrepancy which was also observed in other studies [51-53], could be due to a different prescribing pattern or differing opinions about inappropriateness between the US and Europe [54,55]. Another publication compared seven explicit criteria of drug inappropriateness in elderly patients from different countries, including the Beers criteria [56]. The only drugs considered inappropriate by all seven criteria were long-acting benzodiazepines and tricyclic antidepressants [56]. Diuretics were listed by four of the seven criteria, mainly as potentially inappropriate in combination with other drugs (e.g. NSAIDs, digoxin) or in patients with a history of gout [57-60].

Importantly, one study revealed that up to $40 \%$ of drug-related ED visits are not correctly diagnosed by Emergency Physicians (EP) in a general ED population [3]. Obviously, DRPs can be difficult to diagnose for EPs, especially when elderly patients present with nonspecific complaints. In this subgroup of patients, the proportion of initially missed DRPs was even higher in our study. A potential reason for this might be that EPs are better at identifying DRPs which relate to the patients' chief complaints as compared to DRPs with symptoms 
unrelated to their chief complaints [7]. However, NSCs were not specifically addressed in that study. The high proportion of DRPs that were not identified in our study strongly supports the hypothesis that non-specific manifestations of DRPs are more likely to be missed on admission than DRPs associated with specific symptoms.

\section{Limitations}

Potential limitations of our study include that this study was conducted at a single urban tertiary care centre in Switzerland. The lack of an external validation sample limits the generalizability of the results. Furthermore, age and gender could not be disguised from the outcome assessors, leaving the possibility of some degree of incorporation bias [31]. Also, the definition of non-specific disease presentation might not be generalizable to other settings. Furthermore, we may have missed some cases of patients with NSC during enrolment (no informed consent given; non-inclusion during ED crowding). However, since the data were obtained in a large sample of consecutive ED patients, our NSC cohort appears stable when comparing the different parts of the BANC study in terms of demographic data, rates of acute morbidity, and mortality rate $[22,23]$.

The results of this study are well in accordance with previous investigations demonstrating that a relevant number of drug-related ED visits are undetected $[3,46]$. Furthermore, the identification of DRPs in patients with non-specific complaints seems to be even more difficult than for a general ED population. However, this study here was not designed to investigate this question, as the proportion of missed DRPs in specific complaints was not recorded. An underestimation of DRPs by the expert panel is possible, especially as the clinical pharmacologist was not involved in outcome assessment of all 633 cases. However, other studies have demonstrated similar frequencies of DRPs in ED patients $[3,46,61]$. Additionally, we did not assess for the individual preventability of DRPs in this exploratory study.

The risk of falls is known to be significantly associated with psychotropic medication, especially for benzodiazepines and other hypnotics, as well as for antiepileptics [62]. However, trauma patients with injurious falls were not included in our study.

\section{Conclusion}

The ED is an environment where medication regimens of elderly patients need to be screened systematically for drug-related problems due to both the frequency and the possible severity of the condition. Patients with nonspecific complaints are known to be at high risk for complications - our findings add DRPs to the long list of possible complications and missed conditions in this population. It is important to realize that the number of medications and treatment with specific drugs or drug classes such as thiazides, benzodiazepines, antidepressants, and anticonvulsants are significantly associated with DRPs, resulting in ED visits with non-specific complaints. Better knowledge of these relationships may help to decrease the frequency of missed DRPs in this patient group.

\section{Competing interests}

The authors declare that they have no competing interests.

\section{Authors' contributions}

CHN, MJR, SM, RB study concept and design. CHN, MJR, SM, ASM acquisition of subjects and data. CHN, MJR, SM, AP, MB, RWK, SK, RB analysis and interpretation of data, and preparation of manuscript. (See section on "Authorship and Duplicate Publication"). All authors read and approved the final manuscript.

\section{Acknowledgments}

The authors would like to thank Andy Schötzau (EUDOX, Basel, Switzerland) for performing the statistics, and all physicians and nurses of the community hospitals, geriatrics, and internal medicine, where most of our ED patients received their follow-up and therapy. Special thanks to Dagmar I Keller MD, Luzia Meier MD, Michael Koller MD for help with outcome assessment. Also, we would like to thank Karen Delport MD for helpful discussions.

\section{Author details}

${ }^{1}$ Department of Emergency Medicine, University Hospital Basel, Basel, Switzerland. ${ }^{2}$ Department of Pharmacology and Toxicology, University Hospital Basel, Basel, Switzerland. ${ }^{3}$ Department of Acute Geriatrics, University Hospital Basel, Basel, Switzerland.

Received: 11 July 2012 Accepted: 24 February 2013

Published: 5 March 2013

\section{References}

1. Flaherty JH, Perry HM 3rd, Lynchard GS, Morley JE: Polypharmacy and hospitalization among older home care patients. J Gerontol A Biol Sci Med Sci 2000, 55(10):M554-M559.

2. Goldberg RM, Mabee J, Chan L, Wong S: Drug-drug and drug-disease interactions in the ED: analysis of a high-risk population. Am J Emerg Med 1996, 14(5):447-450.

3. Hohl CM, Zed PJ, Brubacher JR, Abu-Laban RB, Loewen PS, Purssell RA: Do Emergency Physicians Attribute Drug-Related Emergency Department Visits to Medication-Related Problems? Ann Emerg Med 2010, 55(6):493-502. e494.

4. Queneau P, Bannwarth B, Carpentier F, Guliana J-M, Bouget J, Trombert B, Leverve X, Lapostolle F, Borron SW, Adnet F, et al: Emergency department visits caused by adverse drug events: results of a French survey. Drug Safety 2007, 30(1):81-88.

5. Gurwitz JH, Field TS, Harrold LR, Rothschild J, Debellis K, Seger AC, Cadoret C, Fish LS, Garber L, Kelleher M, et al: Incidence and preventability of adverse drug events among older persons in the ambulatory setting. JAMA: The Journal of the American Medical Association 2003, 289(9):1107-1116.

6. Bednall R, McRobbie D, Hicks A: Identification of medication-related attendances at an A \& E department. J Clin Pharm Ther 2003, 28(1):41-45.

7. Hohl CM, Robitaille C, Lord V, Dankoff J, Colacone A, Pham L, Bérard A, Pépin J, Afilalo M: Emergency physician recognition of adverse drugrelated events in elder patients presenting to an emergency department. Academic Emergency Medicine 2005, 12(3):197-205.

8. Kinirons MT, O'Mahony MS: Drug metabolism and ageing. Br J Clin Pharmacol 2004, 57(5):540-544.

9. Crentsil V, Ricks MO, Xue QL, Fried LP: A pharmacoepidemiologic study of community-dwelling, disabled older women: Factors associated with medication use. Am J Geriatr Pharmacother 2010, 8(3):215-224.

10. Sikdar KC, Dowden J, Alaghehbandan R, MacDonald D, Peter P, Gadag V: Adverse drug reactions in elderly hospitalized patients: a 12-year population-based retrospective cohort study. Ann Pharmacother 2012, 46(7-8):960-971. 
11. ElDesoky ES: Pharmacokinetic-pharmacodynamic crisis in the elderly. Am J Ther 2007, 14(5):488-498.

12. Field TS, Mazor KM, Briesacher B, DeBellis KR, Gurwitz JH: Adverse drug events resulting from patient errors in older adults. J Am Geriatr Soc 2007, 55(2):271-276

13. McLean AJ, Le Couteur DG: Aging biology and geriatric clinical pharmacology. Pharmacol Rev 2004, 56(2):163-184

14. Hohl CM, Robitaille C, Lord V, Dankoff J, Colacone A, Pham L, Berard A Pepin J, Afilalo M: Emergency physician recognition of adverse drugrelated events in elder patients presenting to an emergency department. Academic emergency medicine: official journal of the Society for Academic Emergency Medicine 2005, 12(3):197-205.

15. Howard RL, Avery AJ, Howard PD, Partridge M: Investigation into the reasons for preventable drug related admissions to a medical admissions unit: observational study. Quality and Safety in Health Care 2003, 12(4):280-285.

16. Ventura MT, Laddaga R, Cavallera P, Pugliese P, Tummolo RA, Buquicchio R, Pierucci P, Passalacqua G: Adverse drug reactions as the cause of emergency department admission: focus on the elderly. Immunopharmacol Immunotoxicol 2010, 32(3):426-429.

17. Wierenga PC, Buurman BM, Parlevliet JL, van Munster BC, Smorenburg SM Inouye SK, de Rooij SEJA: Association between acute geriatric syndromes and medication-related hospital admissions. Drugs Aging 2012 29(8):691-699. 610.2165/11632510-000000000-000000000.

18. Huang AR, Mallet $L$, Rochefort CM, Eguale T, Buckeridge DL, Tamblyn R: Medication-related falls in the elderly: causative factors and preventive strategies. Drugs Aging 2012, 29(5):359-376. 310.2165/11599460-000000000000000000 .

19. Rutschmann OT, Chevalley T, Zumwald C, Luthy C, Vermeulen B, Sarasin FP. Pitfalls in the emergency department triage of frail elderly patients without specific complaints. Swiss Med Wkly 2005, 135(9-10):145-150.

20. Vanpee D, Swine C, Vandenbossche P, Gillet JB: Epidemiological profile of geriatric patients admitted to the emergency department of a university hospital localized in a rural area. Eur J Emerg Med 2001, 8(4):301-304.

21. Grossmann FF, Zumbrunn T, Frauchiger A, Delport K, Bingisser R, Nickel CH: At risk of undertriage? Testing the performance and accuracy of the emergency severity index in older emergency department patients. Ann Emerg Med 2012, 60(3):317-325. e313.

22. Nemec M, Koller MT, Nickel CH, Maile S, Winterhalder C, Karrer C, Laifer G, Bingisser R: Patients presenting to the emergency department with nonspecific complaints: the Basel Non-specific Complaints (BANC) study. Academic emergency medicine: official journal of the Society for Academic Emergency Medicine 2010, 17(3):284-292

23. Nickel $\mathrm{CH}$, Ruedinger J, Misch F, Blume K, Maile S, Schulte J, Kohrle J, Hartmann O, Giersdorf S, Bingisser R: Copeptin and peroxiredoxin-4 independently predict mortality in patients with nonspecific complaints presenting to the emergency department. Academic emergency medicine: official journal of the Society for Academic Emergency Medicine 2011, 18(8):851-859.

24. Aminzadeh F, Dalziel WB: Older adults in the emergency department: a systematic review of patterns of use, adverse outcomes, and effectiveness of interventions. Ann Emerg Med 2002, 39(3):238-247.

25. Samaras N, Chevalley T, Samaras D, Gold G: Older patients in the emergency department: a review. Ann Emerg Med 2010, 56(3):261-269.

26. Chew WM, Birnbaumer DM: Evaluation of the elderly patient with weakness: an evidence based approach. Emerg Med Clin North Am 1999, 17(1):265-278. $x$

27. Nickel $\mathrm{CH}$, Nemec M, Bingisser R: Weakness as presenting symptom in the emergency department. Swiss Med Wkly 2009, 139(17-18):271-272.

28. Carpenter CR, Heard K, Wilber S, Ginde AA, Stiffler K, Gerson LW, Wenger NS, Miller DK, Society for Academic Emergency Medicine Geriatric Task F: Research priorities for high-quality geriatric emergency care: medication management, screening, and prevention and functional assessment. Acad Emerg Med 2011, 18(6):644-654

29. Hogan TM, Losman ED, Carpenter CR, Sauvigne K, Irmiter C, Emanuel L, Leipzig RM: Development of geriatric competencies for emergency medicine residents using an expert consensus process. Acad Emerg Med 2010, 17(3):316-324

30. Sikdar KC, Alaghehbandan R, MacDonald D, Barrett B, Collins KD, Donnan J, Gadag V: Adverse drug events in adult patients leading to emergency department visits. Ann Pharmacother 2010, 44(4):641-649.
31. Knottnerus JA BF: The evidence base of clinical diagnosis: theory and methods of diagnostic research. London, England: Wiley; 2008.

32. Gilboy NTP, Travers DA (Eds): Emergency severity index, version 4: implementation handbook. Rockville: Agency for Healthcare Research and Quality; 2005.

33. Grossmann FF, Nickel CH, Christ M, Schneider K, Spirig R, Bingisser R: Transporting clinical tools to new settings: cultural adaptation and validation of the Emergency Severity Index in German. Ann Emerg Med 2011, 57(3):257-264

34. Safwenberg U, Terent A, Lind L: The Emergency Department presenting complaint as predictor of in-hospital fatality. Eur J Emerg Med 2007, 14(6):324-331.

35. Elmstahl S, Wahlfrid C: Increased medical attention needed for frail elderly initially admitted to the emergency department for lack of community support. Aging (Milano) 1999, 11(1):56-60.

36. Charlson ME, Pompei $P$, Ales KL, MacKenzie CR: A new method of classifying prognostic comorbidity in longitudinal studies: development and validation. J Chronic Dis 1987, 40(5):373-383.

37. Fick DM, Cooper JW, Wade WE, Waller JL, Maclean JR, Beers MH: Updating the beers criteria for potentially inappropriate medication Use in older adults: results of a US consensus panel of experts. Arch Intern Med 2003, 163(22):2716-2724.

38. American Geriatrics Society Beers Criteria Update Expert P: American geriatrics society updated beers criteria for potentially inappropriate medication use in older adults. J Am Geriatr Soc 2012, 60(4):616-631.

39. Thomson: MICROMEDEX. Available at: http://www micromedex.com. Accessed Jan 2003

40. Foundation Pharmaceutical Care Network Europe (PCNE) (Ed): PCNEclassification of drug-related problems V 5.01. 2006. Available at http://www. pcne.org.

41. Schaefer M: Discussing basic principles for a coding system of drugrelated problems: the case of PI-Doc ${ }^{\circledR}$. Pharmacy World \&amp; Science 2002, 24(4):120-127.

42. Lampert ML, Kraehenbuehl S, Hug BL: Drug-related problems: evaluation of a classification system in the daily practice of a Swiss University Hospital. Pharm World Sci 2008, 30(6):768-776

43. Bushardt RL, Massey EB, Simpson TW, Ariail JC, Simpson KN: Polypharmacy: misleading, but manageable. Clin Interv Aging 2008, 3(2):383-389.

44. Levey AS, Bosch JP, Lewis JB, Greene T, Rogers N, Roth D: A more accurate method to estimate glomerular filtration rate from serum creatinine: a new prediction equation. Modification of diet in renal disease study group. Ann Intern Med 1999, 130(6):461-470.

45. Naughton CDJ, Treacy P, Fealy G, Kilkenny M, Johnson F, Butler M: How different are older people discharged from emergency departments compared with those admitted to hospital? European Journal of Emergency Medicine 2011, 18:19-24.

46. Hohl CM, Dankoff J, Colacone A, Afilalo M: Polypharmacy, adverse drug related events, and potential adverse drug interactions in elderly patients presenting to an emergency department. Annals of Emergency Medicine 2001, 38(6):666-671.

47. Hastings SN, Schmader KE, Sloane RJ, Weinberger M, Goldberg KC, Oddone EZ: Adverse health outcomes after discharge from the emergency department-incidence and risk factors in a veteran population. $J$ Gen Intern Med 2007, 22(11):1527-1531.

48. Fick DM, Mion LC, Beers MHJLW: Health outcomes associated with potentially inappropriate medication use in older adults. Res Nurs Health 2008, 31(1):42-51.

49. Schuler J, Duckelmann C, Beindl W, Prinz E, Michalski T, Pichler M: Polypharmacy and inappropriate prescribing in elderly internal-medicine patients in Austria. Wien Klin Wochenschr 2008, 120(23-24):733-741.

50. Budnitz DS, Lovegrove MC, Shehab N, Richards CL: Emergency hospitalizations for adverse drug events in older Americans. The New England journal of medicine 2011, 365(21):2002-2012.

51. Somers A, Robays H, Vander Stichele R, Van Maele G, Bogaert M, Petrovic M: Contribution of drug related problems to hospital admission in the elderly. J Nutr Health Aging 2010, 14(6):477-482.

52. Onder G, Landi F, Liperoti R, Fialova D, Gambassi G, Bernabei R: Impact of inappropriate drug use among hospitalized older adults. European Journal of Clinical Pharmacology 2005, 61(5):453-459.

53. Budnitz DS, Shehab N, Kegler SR, Richards CL: Medication Use leading to emergency department visits for adverse drug events in older adults. Annals of Internal Medicine 2007, 147(11):755-765. 
54. Fialová D, Topinková E, Gambassi G, Finne-Soveri H, Jónsson PV, Carpenter I, Schroll M, Onder G, Sorbye LW, Wagner C, et al: Potentially inappropriate medication use among elderly home care patients in Europe. JAMA: The Journal of the American Medical Association 2005, 293(11):1348-1358.

55. Gurwitz JH, Rochon P: Improving the quality of medication use in elderly patients: a Not-So-simple prescription. Arch Intern Med 2002, 162(15):1670-1672.

56. Chang CB, Chan DC: Comparison of published explicit criteria for potentially inappropriate medications in older adults. Drugs Aging 2010, 27(12):947-957.

57. Rognstad S, Brekke M, Fetveit A, Spigset O, Wyller TB, Straand J: The Norwegian General Practice (NORGEP) criteria for assessing potentially inappropriate prescriptions to elderly patients. Scandinavian Journal of Primary Health Care 2009, 27(3):153-159.

58. McLeod PJ, Huang AR, Tamblyn RM, Gayton DC: Defining inappropriate practices in prescribing for elderly people: a national consensus panel. CMAJ 1997, 156(3):385-391.

59. Gallagher PRC, Byrne S, Kennedy J, O'Mahony D: STOPP (Screening Tool of Older Person's Prescriptions) and START (Screening Tool to Alert doctors to Right Treatment). Consensus validation. Int I Clin Pharmacol Ther 2008, 46(2):72-83.

60. Winit-Watjana W, Sakulrat P, Kespichayawattana J: Criteria for high-risk medication use in Thai older patients. Archives of Gerontology and Geriatrics 2008, 47(1):35-51.

61. Sikdar KC, Alaghehbandan R, MacDonald D, Barrett B, Collins KD, Donnan J, Gadag V: Adverse drug events in adult patients leading to emergency department visits. Ann Pharmacother, 44(4):641-649.

62. Modreker MK, von Renteln-Kruse W: Medication and falls in old age. Internist (Berl) 2009, 50(4):493-500.

doi:10.1186/1757-7241-21-15

Cite this article as: Nickel et al:: Drug - related emergency department visits by elderly patients presenting with non-specific complaints.

Scandinavian Journal of Trauma, Resuscitation and Emergency Medicine 2013 21:15.

\section{Submit your next manuscript to BioMed Central and take full advantage of:}

- Convenient online submission

- Thorough peer review

- No space constraints or color figure charges

- Immediate publication on acceptance

- Inclusion in PubMed, CAS, Scopus and Google Scholar

- Research which is freely available for redistribution 